\title{
Mesenquimomas en la infancia
}

Stout en el año 1948 , inició unáa serie de trabajos en los cuales liegó a la definición gencrica de mesenquimoma, cilificánrlalo cono un tumor de partes blandias, compuesto por dos o más clementos mesenquimáticos que no $\mathrm{sc}$ encuentran juntos ordinarianente, en un neoplasma. Se exceptúa de este criterio la presencia de elcmentos fibrosos. Se liá encontrido: hueso, cartilago, grasa, vasos sanguíneos, musculatura lisa y estriada, tejiclo mixomatoso, linfoílco y henatopoyctico $(1,2,3,4,5)$.

Hay valias publicitciones sin embargo, cn las cuales se tiende a denominar al tumor de acuerclo al tejido que muestan predominio histológico, como jor ejemplo: Angiomioliporna, bamartoma mesenquimático, rabdomiosarcoma, etc. $(6,7,8,9)$. Esta situación puclicra ser desorientadora por cuanto un análisis más detallaclo de los tejidos componentes, como par ejemplo el estudio histoquimico, potría no permitir tal diferencia.

\section{MATERIAI Y METOIDO}

Se induyen los casos diagnosticatos como mesencuimoma benigno o maligno en el Servicio de Anatomía Patológica del Hospital Luis Calvo Mackenna, en los viltimos 5 años (1970. 1975)

Se analizó la edad, localización del tumor. sexo, sinlomas en la primera consulta, trata-

\footnotetext{
*Hospital Calvo Mackenna.

* Tecnólogos Médicos, Servicio de Anatonia Patolingica, Hospital Catwo Mackenna.
}

Rev. Chilena de Pedittria, vol, 47. No $1,1 \leqq 70$
Drs. Rolecto Joklorkovsky*, J. J. Latorre*, V. Beresi* y Sras. Natry Camrona*" y Edkła Diaz"*.

miento efectuado, parámetros de laboratorio, y sobrevida de todos los casos.

Se procedió a tabular semis uantitativamenle diferentes tejiclos mesentuimatosos que se evidenciaron al laker tinciones especificas para musculatura estriada y lisa mediante el mé todo fosfotúngstico de Mallory (PTH); para reticulina, tinciones con el método argéntico cle Gomory, y mucopolisacáridos nentros con la tinción con ácido peryódico de Shiff (PAS). En este último caso, como el método también tiñe glicógeno, cada preparación se analizó con sut control respectivo de diastasa (10). No st din̆eron grasis.

\section{RESUITADOS Y COMENTARIO}

El criterio histológico de mesenquimoma maligno to purlemos resumir en los siguientes puntos: a) tejido embrionario, b) multifacéico of vriabilidad estrutcual y morfológita clular (11, 12).

Los clatos clinicos cstán resumidos en las tablas la y lb.

E1 material clínico consiste en 6 niños on mes nquimomas benignos y otros 6 con malignos. Las edades en el monento del diagnósti. co, lluctúan entre 2 y 12 años para los maliognos, y entre 1 mes y' 10 años para los benignos. Hay 4 niñas entre los malignos y una entre los benignos, en comparación con 2 y 5 niūos respectivamente. En relación al tiempo de evolución antes de la primera consulta, se observa que en los malignos, 8 niños llevaban menos de 2 mescs, uno 1 año, y llama la aten- 
TABLA 1-a

Mesenquimomas malignos

\begin{tabular}{|c|c|c|c|c|c|c|c|c|c|}
\hline$A^{10}$ & Edod & Sexo & $\begin{array}{l}\text { Tiempo } \\
\text { evolusión }\end{array}$ & Sintomas & Locofización & vits & $\begin{array}{c}\text { Tóme } \\
H b\end{array}$ & H! & Sobrevido \\
\hline 7 & $20 \overline{o s}$ & $M$ & 2 meses & urinarios & vegigo & 50 & $H g r$ & $33 \%$ & 32 meses \\
\hline 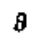 & 4 oños & $F$ & 2 meses & putmonor & mediositino & 39 & 9.5 & 29 & 18 meses \\
\hline 9 & 8 años & $M$ & Sines & nosut & rinoforinx & 120 & 7.5 & 21 & 7 moses \\
\hline 10 & 20 aัตs & $F$ & 1 oño & puivies & vitiva & $?$ & 144 & 49 & desconocido \\
\hline$y$ & 5 oñcs & $F$ & 7.5 oñes & onct inguinat & ans inguinal & 65 & 114 & 34 & 36 meses + \\
\hline 12 & 12 años & $F$ & 1.5 อกัก & nasol & rinofarinx & 34 & $16 k$ & 42 & $\begin{array}{l}38 \text { mesest } \\
+=\text { foileciotos }\end{array}$ \\
\hline
\end{tabular}

TABLA 1-D

Mesenquimomas benignos

\begin{tabular}{|c|c|c|c|c|c|c|c|}
\hline \multirow[b]{2}{*}{4} & \multirow[b]{2}{*}{ Edad } & \multirow[b]{2}{*}{ Sers } & \multirow{2}{*}{\multicolumn{2}{|c|}{ therpoo woiltion }} & \multirow[b]{2}{*}{ Leccise or sein } & \multicolumn{2}{|c|}{ Examenes 5} \\
\hline & & & & & & HD & Mr \\
\hline 1 & 1 mes & $H$ & 15 & nes & tose nosoi & $\alpha 2$ & is \\
\hline 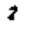 & 100 ofos & 5 & 2 & orips & Jeso imano & 16 & 42 \\
\hline J & 75 mes & $M$ & ? & $?$ & rupro cabetiudo & 143 & 36 \\
\hline 6 & 15 mes & $M$ & 15, & mes & 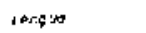 & 125 & 38 \\
\hline 5 & 100 fos & is & & $?$ & Jisotering & 7 & 3 \\
\hline$s$ & 6 meses & $M$ & 1. & meses & tuperd cobetiodo & 17 & 38 \\
\hline
\end{tabular}

ción que 2 casos, 38 meses antes del diagnóstico, habían presentado en la misma zona anatómica un tumor que fue extirpado y catalo gado como benigno en cada oportunidasl.

En los benignos, la mayoría se cliagnosticó antes de 6 meses, a excepción de un niño en que esto ocurrió después de 2 años. El único signo clinico presente fue un aumento de vo. Iumen del tumor.

Todos los mesenquimomas estudiados presentaron una ubicación anatómica variable y asimisnto todos dieron síntomas en relación al órgano alectado. En el caso $7^{\circ}$ el tumor se localizó en vejiga, y el motivo de consulta fueron molestias urinarias y hematuria, El caso 8 presentó un tumor de mediastino y consul. tó por una neumopatía crónica catalogada como empiema inicialmente primero y pos!ejiormente como quiste hidatídico. El 9 9 era un tumor de rinofarinx, y que curso con una rinorrea inespecífica. El 129 también se ubicó en rinofarinx $y$ se presentó con una rinorrea y olsstrucción nasal crónica dle más de un año y medio de evolucion. En este caso como se comentó, se habia hecho una extirpación cle pólipos benignos 18 meses antes. En el caso 109 el mesenquimoma estaba localizado en vulva y produjo prurito local. Finalmente el $11^{\circ}$ caso, 18 meses antes de la consulta habia presentado un granuloma perianal extirpado c informado como benigno, pero que aIgunos meses más tarlé recidivó, motivando la consulta en nuestro Hospital.

Todos los casos en el momento de la consulta presentaban un buen estado nutritivo. A excepción del caso número 1 en el que no se cleterminó, Jos malignos presentaron velocidad de sedimentación elevada (sobre 40), Dos de los 6 niños ya en su primera consulta tenian hemoglobina bajo $10 \mathrm{gr} . \%$.

En ninguno de los benignos se detectó anemia.

Todos los niños cont tumores benignos recibieron tratamiento quirúrgico y no han acusado problemas posteriores en relación a su tu. 
nor. Los niños con mesenquimomas malignos fueron tratados con cirugía, radiotesapia, y quimioterapias variables de acuerdo al caso individual.

La solbrevida es la que se anota en la tabla. Sólo dos niños sobrevivieron; 32 y 19 meses respectivamente, en estado de remisión clínica y con indices de laboratorio aceptables; $3 \mathrm{ca}$ - sos fallecieron clespués de $\pi, 36$, y 38 meses de tratamiento respectivamente. Del caso número 4 se desconoce su evolución.

El análisis histopatológico está resumido en las tablas 2 y 3 . En los mesenquimomas benignos se ve que todos tienen músculo liso, reticulina y mucopolisacáridos neutros. Musculo estriado se observó en 2 casos. El caso nú.

TABLA 2

BENIGNOS

\begin{tabular}{|c|c|c|c|c|}
\hline cosos & $\begin{array}{l}\text { Musc. } \\
\text { liso }\end{array}$ & $\begin{array}{l}\text { Musc } \\
\text { estriodo }\end{array}$ & $\begin{array}{c}\text { Fibras } \\
\text { reticulino }\end{array}$ & $\begin{array}{c}\text { Mucopotisocóridos } \\
\text { neutros }\end{array}$ \\
\hline 1 & ++ & + & ++ & +++ \\
2 & ++ & - & ++-+++ & +++ \\
3 & ++ & + & ++ & +++ \\
4 & ++ & + & +++++ & +++++ \\
++ & + & +++ & +++++++ \\
\hline
\end{tabular}

TABLA 3

\section{MALIGNOS}

\begin{tabular}{|c|c|c|c|c|}
\hline Cosos & $\begin{array}{l}\text { Musc } \\
\text { liso }\end{array}$ & $\begin{array}{l}\text { Musc. } \\
\text { estriodo }\end{array}$ & $\begin{array}{l}\text { Fibras } \\
\text { reticulino }\end{array}$ & $\begin{array}{c}\text { Mucopolisacaridos } \\
\text { neutros }\end{array}$ \\
\hline 7 & ++ & - & $t+-t+$ & $++t$ \\
\hline 8 & ++ & + & $++-t+$ & +++ \\
\hline 9 & ++ & - & +-++ & $++-++t$ \\
\hline 10 & ++ & $+t+$ & $+t$ & $+t+-t++t$ \\
\hline 11 & ++ & + & $++-t+t$ & +-++ \\
\hline 12 & ++ & - & $+-t+$ & ++ \\
\hline
\end{tabular}


mero 6 no se pudo evaluar descde el punto de vista cle los inucopolisacáridos.

Para los mesenquimomas malignos, el análisis de músculo liso, reticulinat y mucopolisacáridos no varia sensiblemente en relación a lo que se observó en los benignos. Músculo estriado se observa en 3 casos.

A nuestro parecer $y$ en relación con los hallazgos histológicos, podemos comentar que en los tumores de origen mesenquimático raramente predomina algún tipo de tejido a excepción del caso número 10 en el cual des:aca la existencia de estriaciones citoplasmáticas celulares que bien podrían corresponder a un mesenquimoma catalogado como rabdomiosar. coma anaplásico, (15) (ver Fig. No 1). Sin cmbargo en este caso se cumplen los principios enunciados al comienzo, vale decir la existencia de 20 más elementos mesenquimáticos que no se encuentran frecuentemente juntos en un neoplasma, lin la Fig. No 2 teñida con técnicas histológicas corrientes (hematoxilina-eosjna), no es posible clistinguir claramente las estriaciones citoplasmáticas ni la proporción de mucopolisacáridos, elementos que se destacan con las tinciones de PTH y PAS respectiva. mente. Requisito fundamental en la última es la contraprueba con diastasa que elimina el glicógeno teñido por el ácido peryódico (Figuras 3 y 4 ).

Otro elemento encontrado en todos los casos analizados es la existencia de fibras de reticulina que se ven con el método de Gomori, lsto se demuestra claramente en la figura No 5 ; la fibra reticular es la menos evoluciona. da de todas liss fibras orgánicas y es por esta razón que se presenta en tal cantidad en las estructuras mesenquimáticas analizadas ya sean benignas o malignas.

La figura $\mathrm{N}^{0} 6$ demuestra músculo liso que fácilmente se destaca con la técnica de PTH, siendo este hallazgo el más uniformemente encontrado en todos los casos.

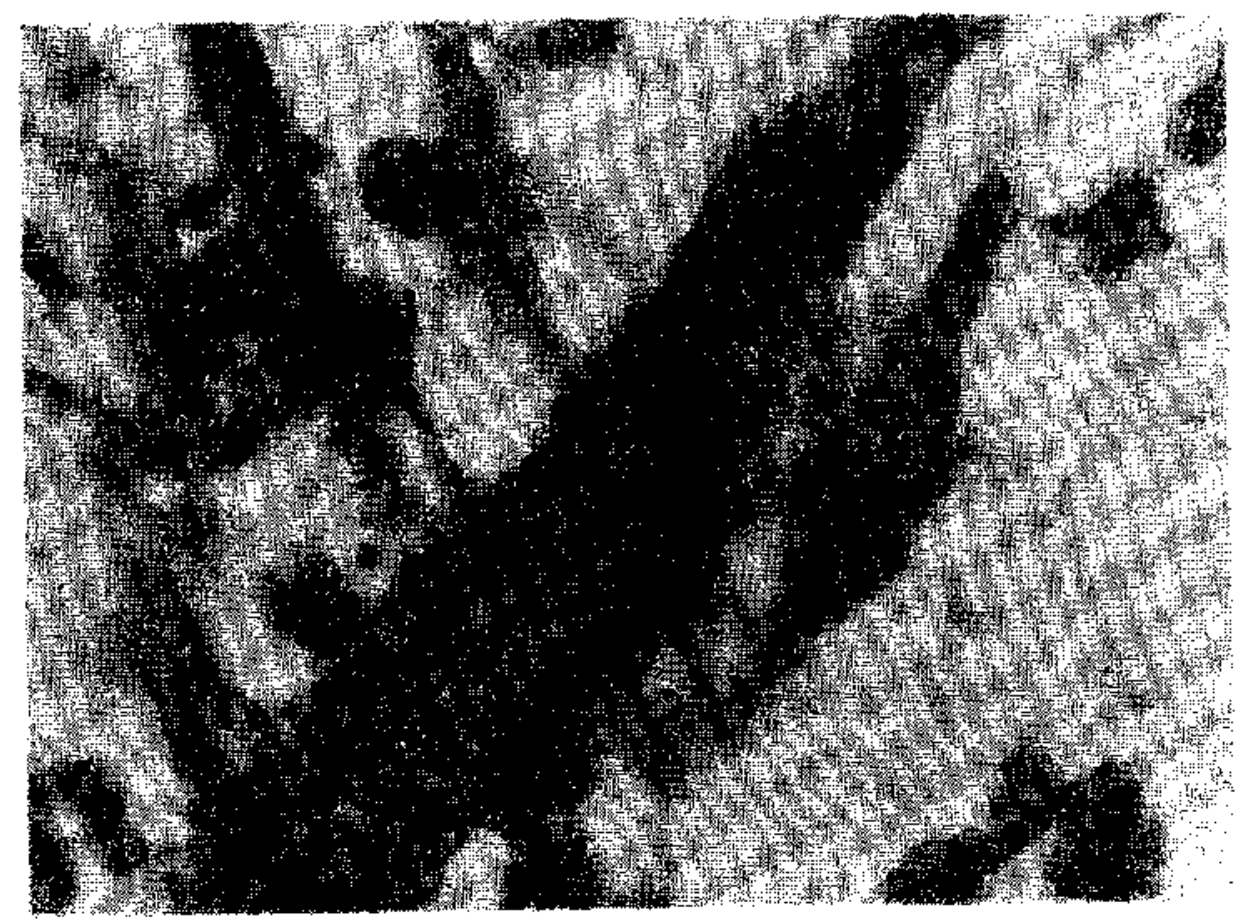

Figura $\mathrm{N}^{0} 1-\mathrm{T}$ inción PTH (PL $40 \times 10$ ). Pernite destacar estriaciones ciloplasmáticas y fibritares. 


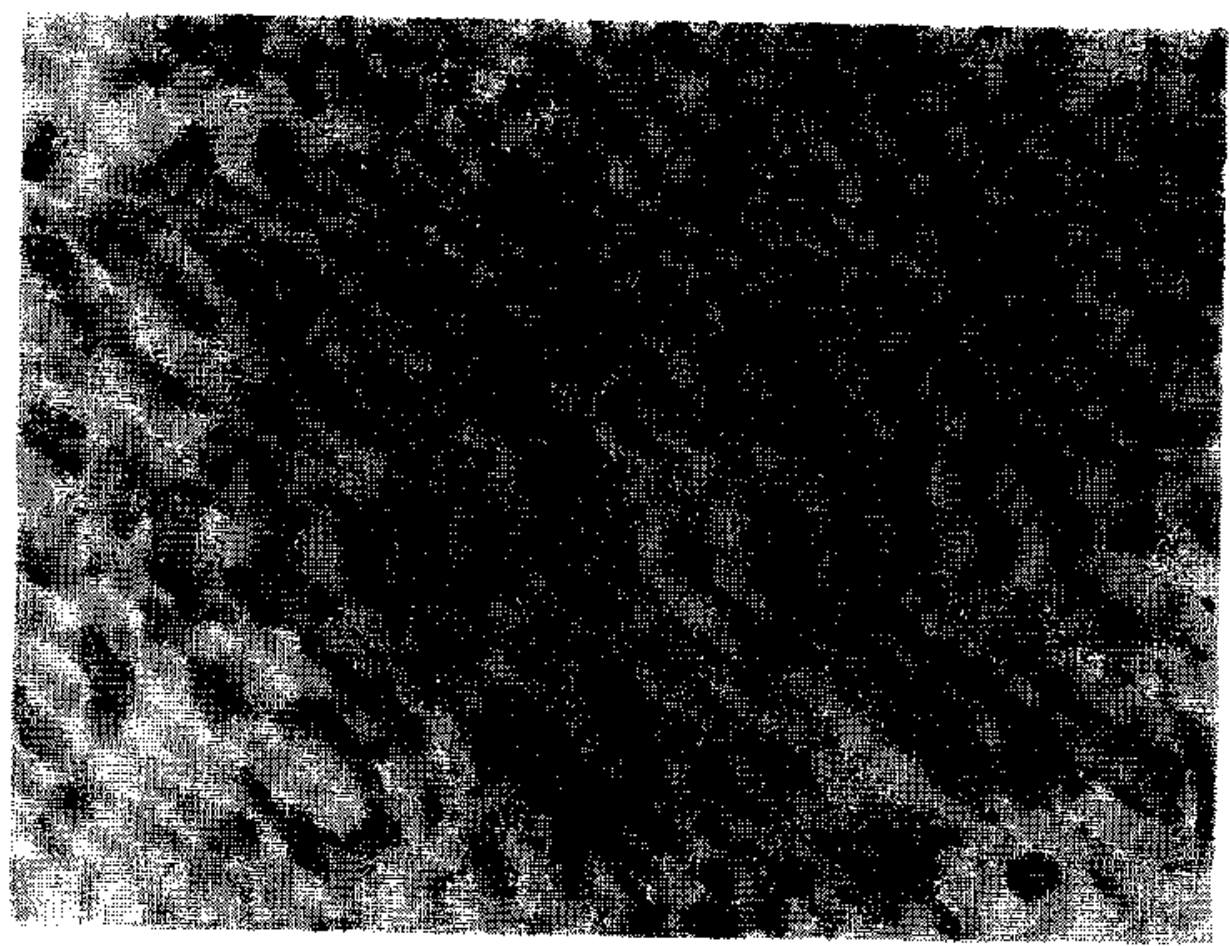

Figura $\mathrm{N}^{2}-$ Tinción $\mathrm{HF},\left\langle\mathrm{l}^{\circ} \mathrm{I}, 40 \times 10\right\rangle$. No permite diferetriar las fibras nusculares estriadas Sólo evidencia gran variabilidad celujar y anapiasia.

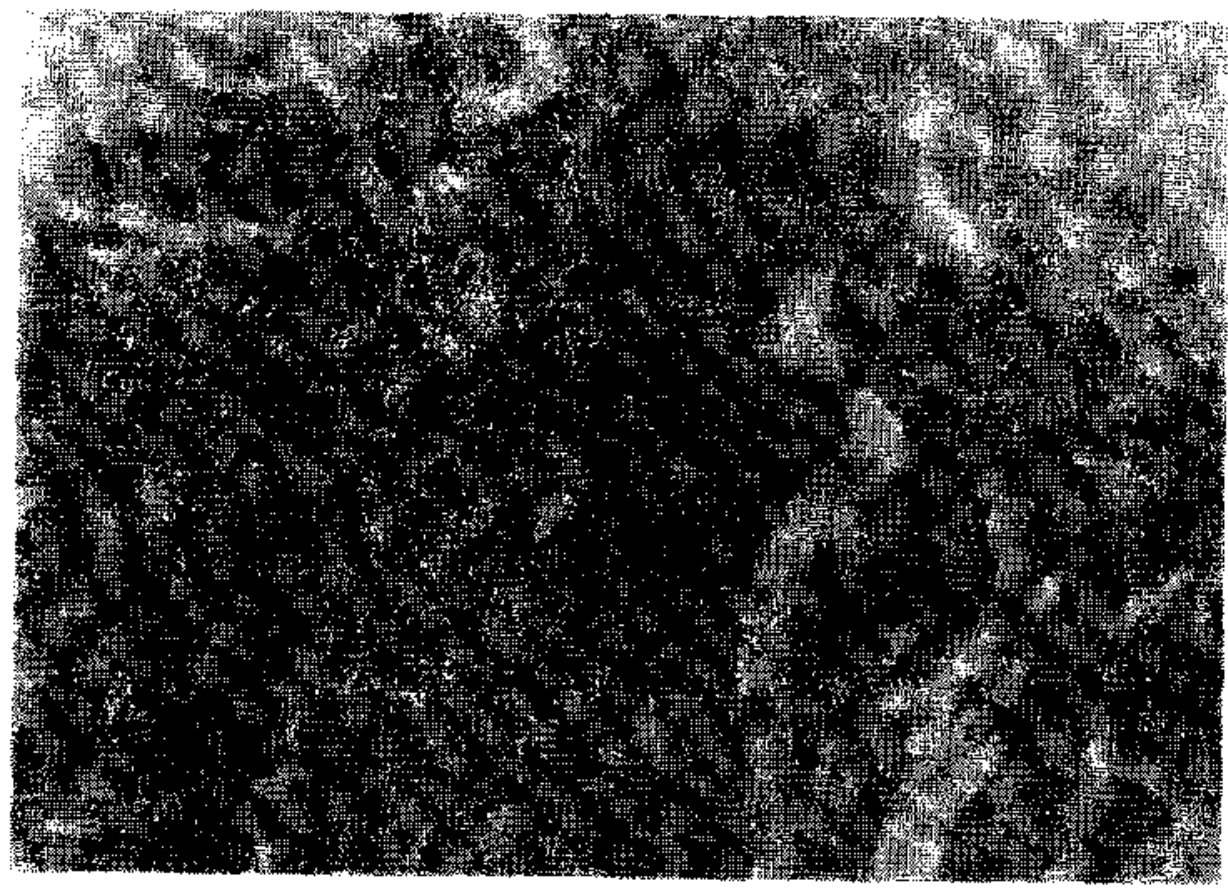
Figura No 3 - PAS (PL $40 \times$ I0). Demuestra la existencia de mucopolisacíridos difusamente
distribuidos en el espesor do la masa tumoral. 


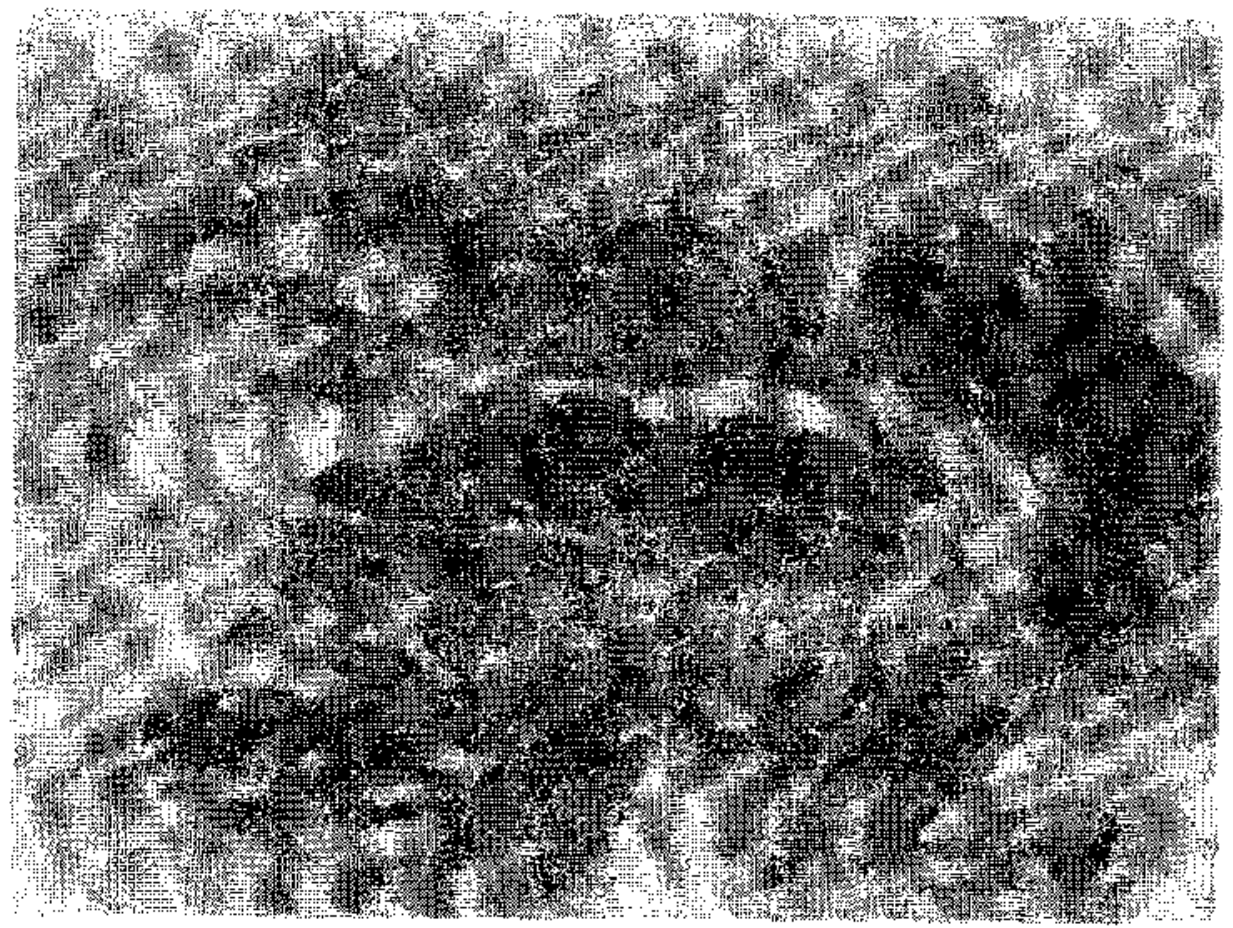

Figura No 4 - Diastasa PAS. Técnica complenentatin de la anterior no detuestra variaciones tintoriales.

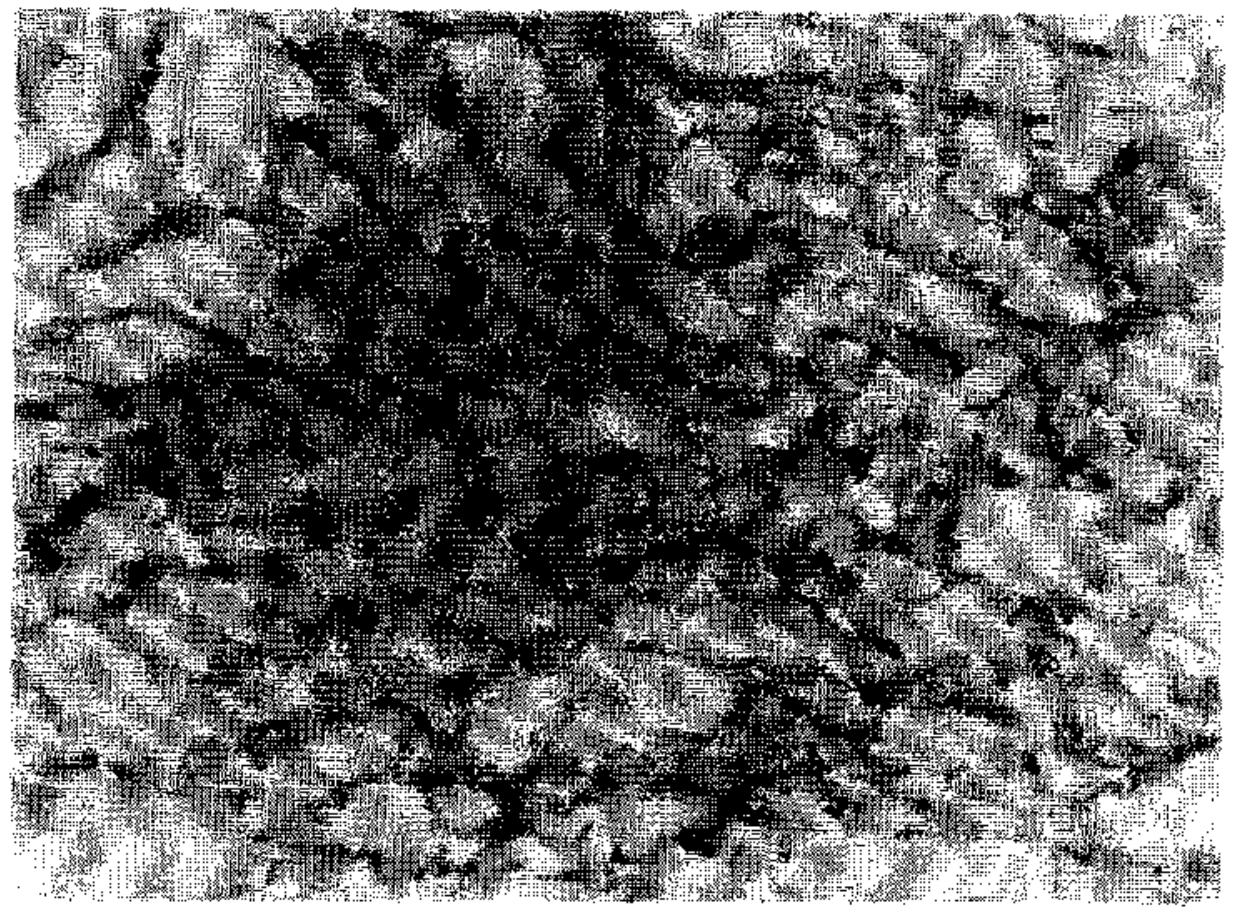

Figura No 5 - Método de Gomori (IML 40 $\times$ 10). Denluestra proliferación difusa de fibra reticular. 


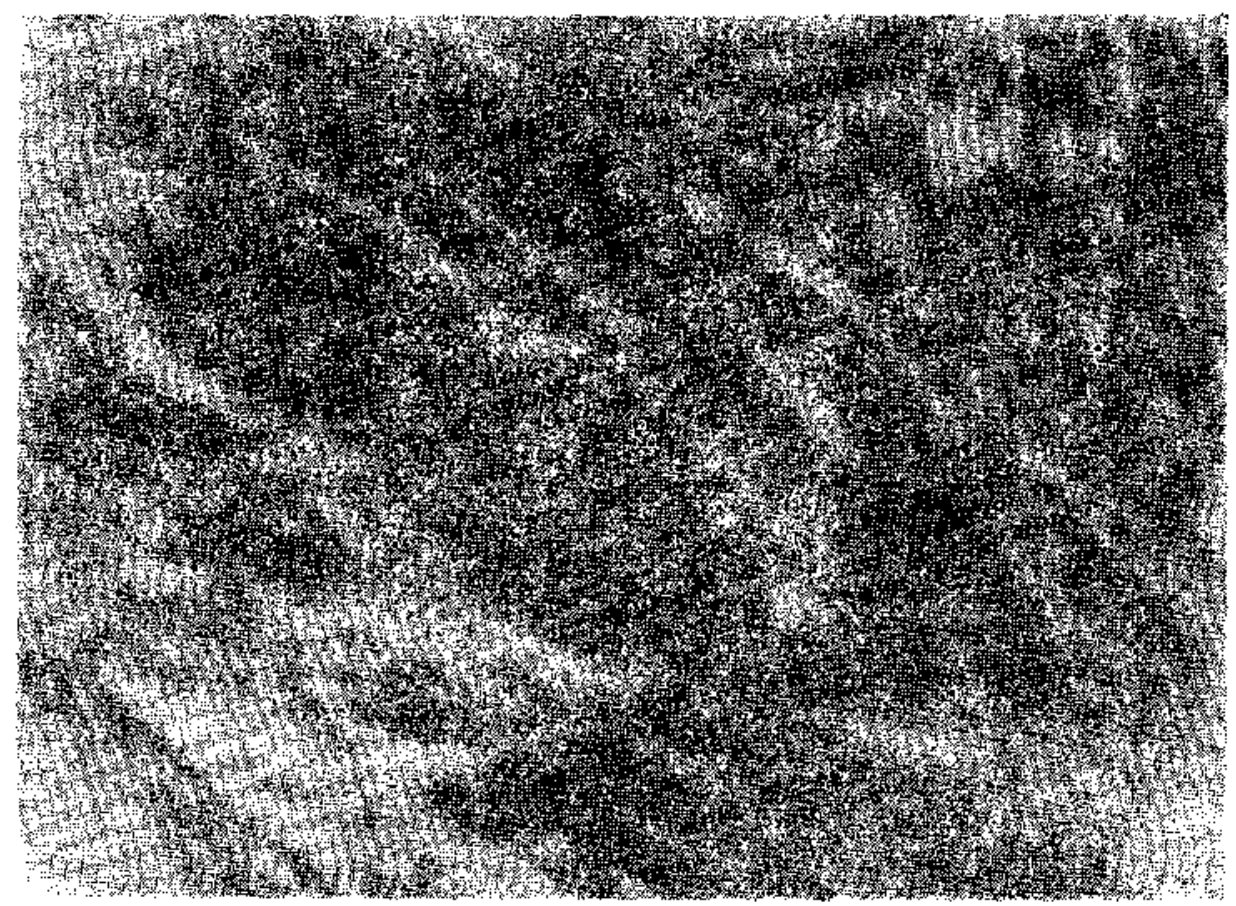

Figura No $6-$ Mitolo PTH (PI. $40 \times 10$ ). Permitc alomosime músculo liso.

\section{CONCLUSIONES}

La predominancia de algún tipo de tejido especílico no es un hecho corriente en los mesenquumomas. Por el contrario, utilizando técnicas listoquímicas específicas, se ha denostrado en el presente trabajo, Ia existencia de variadas estructuras tistulares que de por sí no confieren una distinción tumoral conocida, como seria el caso de lejomioma, liposarcoma, sarcoma botriodes, elc. Por consiguiente el tér. mino mesenquimomas es apropiado a lit patología turnoral infantil.

En los mesenquimomas considerados beniy. nos, es posible también observar nultiplicidad de tejiclos pero, estos son mís diferenciados y maduros.

Finalmente, crecmos que las térnicas histoquímicas propuestas deberían ser consideraclas cada vez que se plantea el dingnóstico de mesenquimoma.

\section{SUMARIO}

Se expone la experiencia de los últimos 5 años del Hospital Luis Calvo Mackenna en
12 casos de mesenquimomas, 6 de ellos malig. nos, de los cuales fallecicion 5 , y 6 casos benignos con evolución clínica satisfactoria.

En cada caso se practicaron tinciones histoquimicas con PTiH, PAS, y Gomori, evidencianclose en todos, varias clases de tejidos meseny̧uimáxicos sin predominio de alguno de ellos cn especial, por lo que se concluye que el término mesenquimoma cs $\mathrm{cl}$ más apropiado.

Se propone la incorporación de estas técnicas al análisis sistemítico de este tipo tumoral.

\section{SUMMARY}

The analysis of 12 children with clinical and histopathological diagnosis of mesenchymoma is presented. They correspond to the experience seen in Hospital Calvo Mackenna over a period of five ycars (1970-1975).

Six cases are malignant and 3 of them died, and 6 are beningn with a satisfactory evolution.

Different Iristochemical tccliniques (PTH, PAS, and Gomori) were carried out in each case, and different kinds of mesenchymal tissues were thus identified;; predominance 
of a single tissue was not found. Therefore we conclude that the term mesenchymoma is apropiate for defining this tumor. The above mentioned techniques should be done sistema. tically whenever this diagnosis is suspected.

\section{REFER ENGIS}

1. Stout, A. P.: Mesemchymoma mixed tumor of mesenchymal derivatives; Am. Surg. 127: 278-290, 1948.

2. Hyde, White and Stout: Mesemchymoma of the mesentery. Cancer 3: 653-656, 1950.

3. Stout, Lattes: Tumors of soft tissues: Atlas of tumor pathology; Washington D. C. Armed Forces Istitute of Pathology, 1967 series 2 fascicle 1 pages 1972-1973.

4. Holdsworth: Malignant mesenchymoma in infants: Am. J. Dis. Child. Vol. 128 1974; pages 847-850.
5. Ber and Stout: Benign mesendmomoma in clifidren: Cancer Vol. 15, 1962.

G. Stout: Tumors of bloxd vessels. Texas State J. Mrtl. $40: 362-365$.

7. Brody and Lipsuchtz: Concomitant intrarenal and prerenal angiomyolipoma J. Urol. 74: 741-746, 1965.

8. Perou and Giay: Mesenchymal lamartumas of kidncy, J. Urol. 8s: 240-261, 1960.

9. Pratt: Cancer Res, 32: 606-610. 1972.

10. Lillie: Histopatbological techunic and practical histochemistry Third Edition, 1965.

11. Stowens: I'ediatric Pathology.

12. Robbins: Patologia estrnctural y funcional, 1975.

13. Watern Sutow: Clinical Pediatric Oncology, 1979. 